$16^{\text {th }}$ International Congress of Metrology, 07001 (2013)

DOI: $10.1051 /$ metrology/201307001

(C) Owned by the authors, published by EDP Sciences, 2013

\title{
LA METROLOGIE AU SERVICE DES PATIENTS
}

\author{
Guy CRISTIAN - Christelle STUMPF - Jean-François MORO \\ BIOCORDIS France, 3, rue du Bois Chaland, 91090 LISSES, France \\ +33160756909-info@biocordis.com - www.biocordis.com
}

\begin{abstract}
Résumé. Dans le domaine de la santé, sans que le professionnel voire le patient en ait pleinement conscience, la qualité de fonctionnement des dispositifs médicaux et scientifiques utilisés concourt à la qualité des soins. Pourquoi ? Comment la maîtrise, par les professionnels de la santé, de la fonction métrologie participe à l'évaluation des risques et à leur réduction, à la prévention d'accident ou de mauvais diagnostic ? Des éléments de réponse sont développés par les conférenciers à la lumière de leur expérience au sein d'une entreprise de services dans les domaines de la métrologie et santé.
\end{abstract}

\begin{abstract}
In the field of health, without professional and even the patient has full consciousness, the quality of medical devices used contributes to the quality of care. Why? How the control, by health professionals, of the Metrology concept participates in the assessment of risks and their reduction, prevention of accident or wrong diagnosis? Answers are developed by the speakers in the light of their experience within a service company in the areas of metrology and health.
\end{abstract}

\section{Introduction}

Pour tout acte de soins s'inscrivant dans une démarche préventive, diagnostique, pronostique et thérapeutique, la recherche de la qualité est une des préoccupations essentielles et constantes de tout professionnel de santé, ce quel soit le lieu où il exerce (établissement de santé, laboratoire de biologie médicale, cabinet médical, d'infirmerie ou de kinésithérapie, ...). Parallèlement, dans un monde ouvert et connecté, les patients sont de plus en plus sensibles à la qualité des soins qui leur sont prodigués. Cette double quête vers la qualité se résume par la balance bénéfice-risque, c'est-à-dire la comparaison du risque d'un acte ou d'un traitement à ses éventuels bénéfices, avec des niveaux d'équilibre escomptés qui diffèrent selon les acteurs en présence. - Ces aspects sont d'ailleurs mentionnés très clairement dans les recommandations de l'ARS et de l'HAS, et entrent de façon obligatoire dans le processus d'accréditation des établissements de Santé - Il faut également tenir compte que dans un contexte de plus en plus contraint, complexe, technologique et mouvant, le rapport bénéfice-risque compte de plus en plus de variables. Pour ce qui concerne les facteurs de risque liés à l'acte, au traitement ou au produit, l'équilibre bénéfice-risque est maintenu ou amélioré au prix d'une bonne formation des opérateurs et d'une foule d'éléments méthodologiques. Parmi ces éléments, il en est un qui reste encore sous-exploité, quand il n'est pas ignoré, mais qui concourt à une optimisation de l'équilibre précité : le contrôle et la traçabilité des Performances par la maîtrise de la fonction Métrologie.

Après avoir situé le contexte et les enjeux de santé publique, les auteurs montreront comment la métrologie est présente en filigrane dans la multitude de réglementations et documentations visant à renforcer les dispositifs d'amélioration continue de la qualité et de la sécurité des soins, au bénéfice des patients pris en charge par les établissements de santé et les professionnels exerçant en ville. Les auteurs mettront ensuite en évidence comment la métrologie participe aux activités de maintenance, dont la bonne gestion est essentielle au fonctionnement des établissements de santé. Ils concluront par le rôle significatif bien que non encore généralisé et reconnu comme tel de la métrologie qui doit se renouveler sans cesse pour s'adapter aux évolutions techniques et sociétales et pour accompagner la mutation des soins vers l'e-santé.

\section{Contexte : la gestion des risques associés aux soins, un enjeu de santé publique}

\subsection{Une fréquence élevée d'événements indésirables associés aux soins}

Au cours de la décennie passée, différentes études [1] ont conclu à une fréquence élevée, avec une stabilité des 
constats dans le temps, d'événements indésirables associés aux soins, parfois graves, souvent évitables (on constate en moyenne un tel événement tous les cinq jours dans un service de 30 lits). On trouve là tout l'intérêt, voir la richesse d'enseignement, à généraliser les FEI (fiche d'évènements indésirables) au profit des constructeurs pour suivre la fiabilité des Dispositifs Médicaux, et des responsables Qualité des établissements de soins pour l'amélioration des bonnes pratiques.

Par exemple, un sous-dosage de perfusion peut se produire avec un pousse-seringue automatique du fait de défaut non visible à l'œil nu (comme la déformation de l'axe du piston ce qui sera de toute façon mis en évidence lors d'opération d'étalonnage / vérification-), mais détectable à long terme (plus de $24 \mathrm{~h}$ ) lorsque le personnel soignant s'apercevra de l'injection incomplète de produits dans un temps donné.

Autre exemple : dysfonctionnement/rupture de lève-malade, suite à des coutures affaiblies (du fait de lavages et désinfections répétés, des frottements et de l'usure), à des axes de rotules fatigués pouvant se briser brusquement, ou encore à des crémaillères bloquées en position haute. Une opération de vérification périodique en charge maximale permet de mettre en évidence les dérives anormales, et d'anticiper ces faiblesses.

Cet état n'est pas propre à la France ; il est également partagé au niveau international. L'analyse des causes révèle qu'ils sont dus non pas à un manque de compétence techniques des professionnels mais à des défauts d'organisation, de coordination, de vérification ou de communication. Autrement dit, les dysfonctionnements résultent d'une insuffisance ou d'un manque de culture de sécurité.

Pour y remédier, des démarches de gestion des risques ${ }^{1}$ sont promues par les pouvoirs publics et les professionnels de la santé, avec comme enjeux de santé publique :

- des enjeux humains, concernant à la fois les patients et leurs familles en termes de conséquences émotionnelles et physiques, sociales et familiale, et aussi les professionnels de santé impliqués ;

- des enjeux stratégiques : pérennité de l'établissement / cabinet de santé, réputation, développement d'activités ;

- des enjeux techniques et organisationnels, financiers et juridiques.

Ces démarches visent à concilier prise de risque avec maîtrise des dangers associés. Elles reposent sur l'identification des risques, sur l'élimination de certains risques, sur la prévention et l'anticipation de ceux à prendre pour la prise en charge des patients. Des outils existent déjà : APR et AMDEC.

\footnotetext{
${ }^{1}$ Ces démarches visent à concilier prise de risque avec maîtrise des dangers associés. Elles reposent sur la connaissance des risques, sur l'élimination de certains risques, sur la prévention de ceux à prendre pour la prise en charge des patients.
}

\subsection{Quel périmètre pour les risques associés aux soins}

Dans le domaine de la santé, le risque est encore trop souvent abordé de manière cloisonnée. Certains sont parfaitement pris en compte (par exemple, risque transfusionnel ou infectieux) à la suite ou pas de crises sanitaires fortement médiatisées ; d'autres sont insuffisamment considérés comme la gestion de l'identification du patient ou la maîtrise d'équipements de mesure intervenant directement ou indirectement dans tout processus de soin. Une explication réside en la multitude de catégories de risques, tel qu'illustré par la figure 1, favorisant le parcellement de la gestion des risque au détriment d'une approche globale et coordonnée. Cependant, force est de constater que la survenance d'un événement grave associé aux soins résulte d'un ensemble de causes variées relatives à plusieurs catégories identifiées à la figure 1 et non à une seule catégorie. D'où la nécessité de privilégier une vision globale et interactive.

\subsection{Une démarche collective pour améliorer la sécurité des soins}

De toutes les catégories sus évoquées, nous retiendrons ici celles liée aux activités dites de soutien sans lesquelles les soins ne pourraient être convenablement prodigués et plus particulièrement celle relative aux équipements et à leur maintenance.

La figure 2 illustre comment la maintenance des équipements et la maîtrise des risques associée est déclinée dans un établissement de santé au niveau opérationnel (services biomédicaux) et au niveau utilisateur (personnel soignant ou patient).

\section{Une profusion de cadres réglementaires et recommandations de gestion des risques associés aux soins}

\subsection{Une évolution des mentalités vers une rationalisation de la gestion des risques associés aux soins ...}

Ces dix dernières années ont été caractérisées par une inflation de publication de textes législatifs et réglementaires ou de recommandations en matière de gestion des risques associés aux soins, mais souffrant d'une absence de cohérence d'ensemble. Il a fallu attendre les années 2009 et 2010 pour voir apparaître des dispositions complémentaires visant à rendre cohérentes ces dispositions et à rationaliser la gestion des risques associées aux soins. Citons :

- mise en œuvre de l'accréditation des médecins exerçant une spécialité ou une activité dite "à risque" en établissement de santé ;

- loi dite HPST de juillet 2009 portant réforme de l'hôpital et relative aux patients, à la santé et aux territoires ;

- exigences accrues de la version 2010 de la procédure de certification HAS des établissements de santé en matière 
de gestion des risques et d'évaluation des pratiques professionnelles (EPP);

- émergence de fonctions nouvelles : coordinateur de la gestion des risques associés aux soins, responsables opérationnels de la qualité en radiothérapie, en unité de stérilisation ;

- procédures d'évaluation tierce-partie d'activités médicotechniques :

$\checkmark$ soit obligatoire : accréditation "ISO 15189" de laboratoires de biologie médicale (LBM), accréditation "ISO 17020" d'inspection d'activités comme celles relatives à l'utilisation de rayonnements ionisants ;

$\checkmark$ soit relevant de démarche volontaire : certification d'unités de stérilisation.

Le domaine de la santé poursuit ainsi son évolution en passant d'une approche centrée sur le processus de soins vers une approche globale relative au parcours de soins, de même que les versions 2000 des normes "ISO 9001" et "ISO 17025 " ont introduit en leurs temps une mutation dans la manière de considérer les exigences normatives, d'une approche "procédure / produit" vers une approche "système".

\section{2 ... où la métrologie joue un rôle significatif bien que non identifié comme tel}

Et la métrologie dans tout ça?

Dans de nombreux secteurs technologiques (aéronautique / spatial, nucléaire, automobile, etc.), où les démarches de gestion des risques sont apparues depuis plus longtemps, un des moyens bien identifié et reconnu participant à la réduction desdits risques réside en la mise en place de la fonction métrologie. Ceci englobe tous les aspects bien connus des métrologues : inventaire des instruments de mesure et de contrôle, étalonnage / vérification, traçabilité documentaire et métrologique, définition du mesurande, définition des caractéristiques des équipements de mesure (spécifications d'achat, EMT, ...), estimation d'incertitude, organisation et définition des responsabilités des différents acteurs.

Il en est de même en santé. L'importance de disposer d'équipements aptes à l'emploi est reconnue. Sauf que les aspects métrologiques cités plus haut sont considérés de manière plus ou moins récente et approfondie selon les obligations réglementaires et, dans tous les cas, de manière globale et diluée puisque la fonction métrologie est noyée dans les préoccupations des services de maintenance et dispersée entre les services biomédicaux, techniques, achat, qualité. D'ailleurs la notion de responsable métrologie est quasi inexistante dans les établissements de santé où cette responsabilité est assumée par les ingénieurs biomédicaux, après tant d'autres responsabilités, mais apparaît bien comme telle dans les LBM soumis à l'accréditation "15189". Ainsi, l'obligation réglementaire pousse à la maîtrise de cette fonction métrologie. Citons les cas nombreux de LBM croyant disposer d'équipements fiable pour le stockage et la conservation de produits surgelés ou congelés mais se rendant compte après une caractérisation thermique desdits équipements que leur profil de température n'est pas adapté à leurs besoins.

Toute technologie, tout composant d'un dispositif dérive dans le temps, de par son usure ou ses conditions d'utilisation. La métrologie concourt à la fois à mettre à disposition des informations sur l'état du dispositif et à obliger son utilisateur à en accepter ou non la dérive selon les indications de son analyse de risques.

Ainsi, le sujet de la maintenance des équipements est devenu un sujet d'actualité aujourd'hui dans le domaine de la santé, ce d'autant plus que des textes légaux et réglementaires rendent obligatoire la maintenance de certaines catégories. Pour autant, la mise en place de l'organisation de maintenance dans un établissement / cabinet de santé n'est pas chose aisée, notamment dans les entités ne disposant pas de structure biomédicale.

Avant de poursuivre notre développement en présentant comment mettre en place la maintenance des dispositifs médicaux ou des autres équipements utilisés en santé, il est important de noter que ladite maintenance ${ }^{2}$ est à ne pas confondre avec la matériovigilance ${ }^{3}$. Ces deux activités sont distinctes mais cependant complémentaires. Elles peuvent être réalisées sous la responsabilité d'une même personne ou de deux personnes différentes sous la condition d'une étroite collaboration.

\section{Une bonne gestion de la maintenance est un élément essentiel à la vie d'un établissement de santé, particulièrement dans le cadre de la continuité de fonctionnement des services}

\subsection{Responsabilités des acteurs}

En santé, la responsabilité, pénale (rare) ou civile / administrative (dans ce dernier cas pour les centres hospitaliers publics), est engagée par la victime ou ses proches. Cette responsabilité concerne l'établissement, le personnel médical, l'agent chargé de la maintenance ou des fournisseurs.

En matière de maintenance, elle est exercée par :

- le fabricant ou sous sa responsabilité,

- une société de tierce maintenance, ou

- l'exploitant lui-même.

Dans le premier cas, le matériel est placé sous la responsabilité d'un tiers extérieur à l'établissement. Dans le deuxième cas, le matériel est placé sous la responsabilité du

\footnotetext{
${ }^{2}$ Maintenance : ensemble d'actions permettant de conserver ou de rétablir les fonctionnalités d'un équipement

${ }^{3}$ Matériovigilance : système de surveillance des incidents ou risques d'incidents résultant de l'utilisation de dispositifs médicaux.
} 
tiers mainteneur, mais l'exploitant doit s'assurer que celui-ci apporte des preuves suffisantes de sa compétence. Un des moyens privilégié pour démontrer cette compétence réside en l'accréditation "17025". Enfin, dans le troisième cas, l'exploitant doit recruter du personnel ayant les qualifications nécessaires, lui mettre à disposition des moyens matériels adéquats et le temps requis pour la bonne réalisation des opérations ; dans ce cas, en tant que chef du service de maintenance, un ingénieur biomédical ne saurait s'exonérer de sa responsabilité professionnelle en cas de litige survenant suite à une maintenance déficiente et non liée à une faute professionnelle.

Dans la plupart des centres hospitaliers français, ce sont les deux premiers cas qui se rencontrent (seulement $20 \%$ des exploitants font leur maintenance en interne).

\subsection{Inventaire du matériel concerné}

On distingue deux catégories de matériel :

-les dispositifs médicaux (DM) : Un dispositif médical est un instrument, appareil, équipement ou encore un logiciel destiné, par son fabricant, à être utilisé chez l'homme à des fins, notamment, de diagnostic, de prévention, de contrôle, de traitement, d'atténuation d'une maladie ou d'une blessure. (directive 93/42/CEE relative aux dispositifs médicaux). Les dispositifs médicaux sont répertoriés en quatre classes selon leur niveau de dangerosité. La figure 3 liste de manière non exhaustive des DM courants dans les établissements de santé ;

- les équipements de surveillance et de mesure ${ }^{4}$ : ce matériel permet de mesurer, simuler ou maintenir avec une très grande précision différents paramètres physiques (pressions, débits, volumes d'air ou d'eau, température, hydrométrie, constantes électriques, etc. La figure 4 présente une liste non exhaustive de tels équipements.

S'il est évident pour le patient que le matériel $-\mathrm{cf} .1^{\mathrm{er}}$ point ci-dessus- qui entre en contact direct avec lui ou qui permet d'effectuer des actes diagnostiques soit irréprochable d'un point de vue fonctionnel, il lui est moins évident que d'autres matériels -cf. $2^{\circ}$ point ci-dessus- doivent faire l'objet d'une maintenance irréprochable, d'un suivi permanent, attentif et d'une traçabilité totale. Ces équipements de surveillance et de mesure ne doivent pas être à l'origine d'une mesure fausse, c'est-à-dire en dehors de l'intervalle défini par les EMT $^{5}$ (EMT qui dans la majorité des cas sont les spécifications des constructeurs, mais peuvent être des spécifications réglementaires comme pour les banque de sang ou les autoclaves) ; dans le cas contraire, les conséquences pour les utilisateurs et les patients peuvent se révéler catastrophiques. C'est pourquoi ce matériel est généralement confié à des sociétés de tierce maintenance (comme BIOCORDIS France) possédant des accréditations "17025" dans plusieurs domaines techniques. De plus,

\footnotetext{
${ }^{4}$ Il n'est pas rare de trouver ce matériel sous l'appellation d'ECME (équipement de contrôle, de mesure et d'essai)

${ }^{5}$ Hors laboratoires de biologie médicale qui commencent à être familiarisés, la notion d'incertitude reste assez peu répandue dans les établissements de santé
}

l'utilisation de ce matériel engendre des contraintes fortes de gestion : outres les aspects de traçabilité évoqués plus haut, ce matériel doit être disponible en tout temps. Les sociétés de tierce maintenance sont ainsi amenées à compléter leur offre de service métrologique par des mises à disposition ou location de matériel

Par exemple, l'utilisation d'un pèse-bébé déréglé a pour conséquence de mal déterminer le poids du bébé et donc la dose de produits médicamenteux à lui administrer. Pour les patients en dialyse, où l'on suit entre autres la variation de leurs poids, l'utilisation d'un pèse-personne non répétable et peu sensible, quelle que soit sa justesse par ailleurs, peut mener à des opérations de dialyse incomplète.

\subsection{Analyse des risques}

Quand le fabricant de précise pas de recommandations en matière de maintenance d'un matériel, qu'il soit soumis ou non à obligation de maintenance, l'exploitant n'est pas dispensé de réaliser des contrôles ou des maintenances préventives surtout lorsque ce matériel est soumis à usage intensif ou bien s'il fait partie des équipements vitaux pour le service. Le niveau de sécurité du patient doit rester l'objectif prioritaire.

L'analyse de risque est un des outils permettant de répondre à ce cas de figure. Il n'est pas l'objet de cet article de traiter de méthodes d'évaluation des risques; pour plus de détail, se reporter aux documents [3] et [4] cités en références. Brièvement, l'analyse des risques consiste à identifier les risques, à les caractériser, à les maîtriser ou tout du moins à les réduire par des actions, et à surveiller l'efficacité de ces actions.

Pour ce qui concerne le matériel cité plus haut, l'analyse des risques conclue quasi systématiquement à la nécessité de réaliser périodiquement des opérations de maintenance.

\subsection{Réalisation de la maintenance et Contrôle Qualité}

La maintenance d'un matériel, DM ou équipement de surveillance et de mesure (dans ce dernier cas, il s'agit couramment d'étalonnage / vérification), est l'ensemble des activités destinées à maintenir (maintenance préventive) ou à rétablir (maintenance corrective) ce matériel dans un état ou dans des conditions données de sûreté de fonctionnement pour accomplir une fonction requise (cf. [5]). Les conditions de réalisation de la maintenance sont fixées contractuellement s'il y a lieu entre le fabricant, le fournisseur de tierce maintenance et l'exploitant. Par exemple, lieux de réalisation de la prestation de maintenance : sur le site de l'exploitant ou dans d'autres locaux, ceux du fabricant ou du tiers mainteneur.

En fonction de sa fréquence, de son contenu, du temps à y consacrer, de son coût, de la disponibilité de personnel compétent, la maintenance est réalisée en interne par l'exploitant (par exemple : modifications de paramètres sur moniteurs, remplacement de composants individuels d'usure, 
diagnostic de pannes avec usage d'ECME) ou bien est externalisée (par exemple : étalonnage / vérification desdits ECME, réparation de matériels complexes avec analyse des pannes). À noter qu'externalisation ne signifie pas recours systématique au fabricant ou à une société de tierce maintenance. En effet, différents établissements n'ayant pas le personnel suffisant pour réaliser la maintenance en interne peuvent opter pour une mutualisation de moyens (humains ou matériels).

Toute maintenance, aussi développée soit elle, serait incomplète si elle n'était pas suivie d'un contrôle qualité. En particulier, un contrôle de bon fonctionnement est recommandé avant la remise en service d'un matériel de retour de maintenance, que celle-ci ait été effectuée au sein de l'établissement de santé ou à l'extérieur (envoi dans les locaux d'une société de tierce maintenance).

\section{Conclusion}

Cet article se veut être un aperçu sommaire de la manière dont la métrologie doit être intégrée dans le domaine de la santé via la maintenance des dispositifs médicaux, à l'aide d'équipements de contrôle et de mesure (ECME), maintenance qui est l'un des points fondamentaux de la gestion et la prévention du risque technique. Cette prévention rentre dans le cadre global de la gestion des risques dans les établissements de santé, qui est un volet actuellement essentiel de la politiques hospitalière. In fine, chaque citoyen doit pouvoir avoir accès à une médecine de qualité prouvée. La maîtrise de la fonction métrologie par les acteurs du domaine y participe.
Cependant, une interrogation subsiste : quid de l'e-Santé ? L'e-Santé est un univers rempli d'imagination. Les industriels rivalisent d'idées pour fabriquer les instruments qui faciliteront la vie des patients et des médecins. L'objectif est de permettre aux patients de rester chez eux plutôt que d'aller à l'hôpital ou dans une structure médicalisée. Aujourd'hui, toutes sortes de techniques sont opérationnelles afin de garantir l'autonomie des personnes. En matière de maintenance et de métrologie, tout un pan reste à explorer.

\section{Références}

[1] La sécurité des patients - Mettre en œuvre la gestion des risques associés aux soins en établissement de santé - Des concepts à la pratique, HAS, mars 2012

[2] Maintenance des dispositifs médicaux, Drass MidiPyrénées, 2005

[3] Dispositifs médicaux - Application de la gestion des risques aux dispositifs médicaux, AFNOR, NF EN ISO 14971, Janvier 2013

[4] Maintenance des dispositifs médicaux - Système de management de la qualité pour la maintenance et la gestion des risques associés à l'exploitation des dispositifs médicaux, AFNOR, NF S99-170, Mai 2013

[5] Maintenance hospitalière - Performances des équipements, AFNOR, recueil de normes, Mai 2010

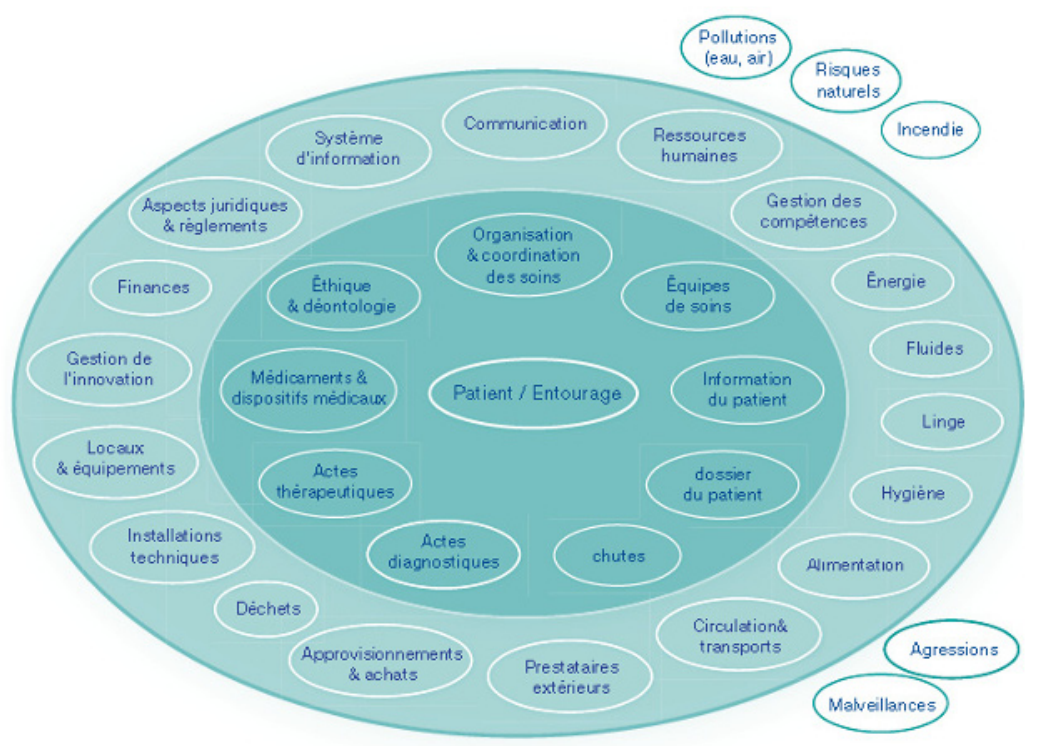

Figure 1 : Approche systématique des grandes catégories de risques en établissement de santé [1] 


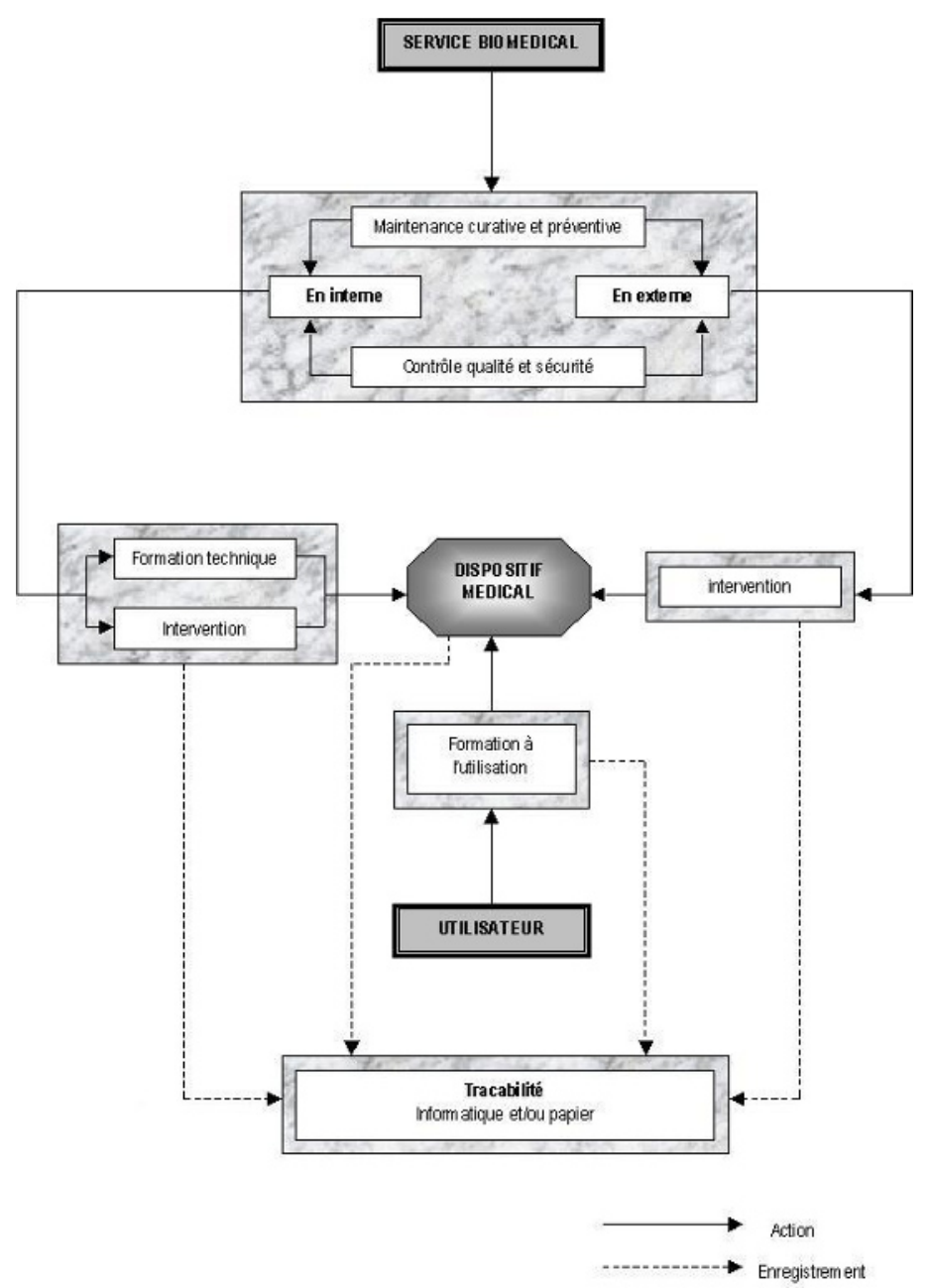

Figure 2 : service biomédical vs maintenance

\begin{tabular}{|c|c|}
\hline DOMAINE & DISPOSITIFS MEDICAUX \\
\hline Chirurgie & $\begin{array}{l}\text { Instrumentation électro-chirurgicale : bistouri, cœlio-chirurgie } \\
\text { Ophtalmologie : laser thérapeutique, phaco-émulsificateur }\end{array}$ \\
\hline Désinfection & Laveur-désinfecteur d'endoscopes \\
\hline Imagerie & $\begin{array}{l}\text { Finalité diagnostique : IRM } \\
\text { Finalité thérapeutique : lithotripteur }\end{array}$ \\
\hline Laboratoire de biologie médicale & Automates, balances de précisions, thermomètres, enceintes climatiques, centrifugeuses, pipettes, ... \\
\hline Pesage* & Systèmes de pesée à visée thérapeutique : pèse-bébé, pèse-personne, pèse-lit, système de pesée sur lève-malade \\
\hline Médecine nucléaire & Réalisation des actes : gamma caméra, PET Scan \\
\hline Monitorage & Moniteurs de suivi patient pour les paramètres vitaux : cardiaque, ventilatoire, système nerveux central \\
\hline Perfusion & Pousse-seringue, pompe à perfusion, PCA (analgésie contrôlée par le patient) \\
\hline Radiodiagnostic & $\begin{array}{l}\text { Production d'images : radiographie ou radioscopie (fixe ou mobile, conventionnelle ou numérisée), scanner, } \\
\text { mammographie, ostéodensitométrie } \\
\text { Injecteur de produit de contraste } \\
\text { Interprétation des images : négatoscope, chaîne numérique }\end{array}$ \\
\hline Radiothérapie & $\begin{array}{l}\text { Délivrance des traitements : radiothérapie, curiethérapie } \\
\text { Console de dosimétrie opérationnelle }\end{array}$ \\
\hline Réchauffement patient & $\begin{array}{l}\text { Table de réanimation néonatale, rampe chauffante, incubateur } \\
\text { Générateur pour couvertures soufflantes ; couvertures électriques }\end{array}$ \\
\hline Stérilisation & Autoclaves \\
\hline Suppléance fonctionnelle & $\begin{array}{l}\text { Rénale : hémodialyse, hémofiltration } \\
\text { Dispositif de traitement et boucle de traitement de l'eau de dialyse } \\
\text { Cardiaque : circulation extra-corporelle, appareil de contrepulsion, stimulateur temporaire, défibrillateur }\end{array}$ \\
\hline Support patient & Table d'opération, lit médicalisé au domicile du patient, lève-patient \\
\hline Ventilation artificielle & $\begin{array}{l}\text { Ventilateur d'anesthésie et de réanimation } \\
\text { Humidificateur chauffant }\end{array}$ \\
\hline
\end{tabular}

Figure 3 : liste non exhaustive de dispositifs médicaux [2] 


\section{$16^{\text {th }}$ International Congress of Metrology}

\begin{tabular}{|l|l|}
\hline CATEGORIE & UTILISATION \\
\hline $\begin{array}{l}\text { Simulateur de patient et testeur de } \\
\text { paramètres } \\
\text { température) }\end{array}$ & $\begin{array}{l}\text { Outils de base du technicien biomédical permettant de vérifier rapidement ou en détail la réponse des } \\
\text { capteurs intégrés dans de très nombreux dispositifs médicaux } \\
\text { Utilisés dans les processus de diagnostic, de révision et de contrôle }\end{array}$ \\
\hline Testeur de sécurité électrique & $\begin{array}{l}\text { Permet de vérifier l'innocuité électrique d'un équipement vis-à-vis du personnel et du patient, par mesure } \\
\text { de courant de fuites qui ne doivent pas dépasser des limites acceptables selon l'utilisation et le degré de } \\
\text { protection de chaque dispositif médical (DM) } \\
\text { Utilisation quasi systématique dans les opérations de maintenance corrective, préventive et de contrôle } \\
\text { qualité, mais aussi en cas de chutes, casses et autres incidents susceptibles de modifier les continuités } \\
\text { électriques des DM }\end{array}$ \\
\hline $\begin{array}{l}\text { Testeur de tensiomètre } \\
\text { Testeur de pousse-seringue } \\
\text { Testeur de pompe volumétrique } \\
\text { Testeur de capteur SpO } 2\end{array}$ & $\begin{array}{l}\text { Ces testeurs permettent de réaliser des cycles de tests complets et automatisés } \\
\text { Utilisation en maintenance corrective, préventive et contrôle qualité. La précision des capteurs de ces } \\
\text { testeurs permet souvent de détecter de façon très précoce la variation d'un paramètre }\end{array}$ \\
\hline $\begin{array}{l}\text { Testeur de défibrillateur } \\
\text { Testeur de bistouri }\end{array}$ & Utilisation ponctuelle pour des campagnes de maintenance préventive et contrôle qualité \\
\hline $\begin{array}{l}\text { Testeur de ventilateur } \\
\text { Fantôme pour la radio ou l'échographie } \\
\text { Testeur de générateur de dialyse } \\
\text { Etc. }\end{array}$ & Équipement destiné à des techniciens "spécialisés" \\
\hline
\end{tabular}

Figure 4 : liste non exhaustive d'équipements de surveillance et de mesure [2] 